\title{
Kant e la Rivoluzione Francese: liberali e/o reazionari fra passione e storia
}

\author{
Kant and the French Revolution: liberals and/or \\ reactionaries between passion and history
}

\begin{abstract}
RIASSUNTO
L'articolo nasce dal tentativo di trovare una connessione all'interno di parte della filosofia kantiana fra i tre termini di "passione", "storia" e "Rivoluzione" (soprattutto francese). La sua intenzione fondamentale è quella di rispondere alle seguenti domande: che ruolo hanno le passioni nel sorgere della storia? E quale significato ha rappresentato la Rivoluzione Francese nel corso delle vicende umane? La ricerca si è occupata principalmente del commento di alcune delle nove tesi del testo kantiano Idea di una storia universale dal punto di vista cosmopolitico ma si è basata anche su altri testi del filosofo di Könisberg, quali Il conflitto delle Facoltà ed i Principi metafisici della dottrina del diritto. Essa si è articolata intorno ai seguenti punti: il ruolo della socievole insocievolezza nel far nascere la società e dunque la storia, il ruolo del popolo nel determinare la storia, la posizione kantiana a favore e poi contro la Rivoluzione Francese. Cercando di determinare se si possa mantenere una linea di sostanziale continuità, nonostante le differenze, nel pensiero kantiano sulla Rivoluzione Francese espresso nei testi sopracitati, l'articolo s'interroga anche sul rapporto fra procedere della ragione nella ricerca della conoscenza e della crescita morale, e filosofia della storia (ragionando particolarmente sul confronto fra l'idea di "fine" della storia e l'uso della ragione, un confronto mediato dal concetto di "organizzazione").
\end{abstract}

Parole chiave: Kant. Passione. Storia. Rivoluzione.

\section{ABSTRACT}

The article comes from an attempt to find a connection, within a part of Kantian philosophy, among the three terms of "passion", "history" and "Revolution" (especially French). The fundamental interest is to answer the following questions:

* Doutora em Filosofia. https://orcid.org/0000-0002-6146-0428 
what role do passions have in the rise of history? And what significance has been the French Revolution representing in the course of human affairs? The research was mainly concerned with the commentary of some of the nine theses of the Kantian text Idea of a universal history from the cosmopolitan point of view but also based on other texts of the philosopher of Könisberg, such as The Faculty conflict and The metaphysical principles of the doctrine of the Laws. It has been structured around the following points: the role of sociable insociability in giving birth to society and therefore history, the role of the people in determining history, the Kantian position in favour and then against the French Revolution. Trying to determine if we can maintain a line of substantial continuity, despite the differences, in the Kantian thought on the French Revolution expressed in the above texts, the article also questions the relationship between the progress of reason in the search for knowledge and moral growth, and philosophy of history (particularly thinking about the comparison between the idea of "purpose" of history and the use of reason, a comparison mediated by the concept of "organization").

Keywords: Kant. Passion. History. Revolution.

\section{La socievole insocievolezza}

La domanda è nata dall'esame del testo Idea di una storia universale dal punto di vista cosmopolitico [1784], ${ }^{1}$ in cui Kant individua il sorgere della storia nella realtà della "socievole insocievolezza" che obbliga l'uomo ad unirsi in una società civile con delle regole. ${ }^{2}$ L'uomo ha delle disposizioni naturali che sono destinate un giorno a svolgersi in modo completo e conforme al loro scopo. Queste naturali disposizioni, dirette all'uso della sua ragione, hanno il loro completo svolgimento solo nella specie, non nell'individuo. La ragione è un "potere di estensione", un potere che - appunto in quanto tale non è attualmente infinito, illimitato - procede per gradi con l'esercizio, imparando a passare lentamente da un grado all'altro della conoscenza. Se poi consideriamo che la vita dell'uomo è per natura breve, proprio da questa gradualità deriva che solo una serie indefinita di generazioni potrà realizzare il pieno sviluppo dei germi insiti nella specie umana.

In questo testo, Kant sintetizza il suo pensiero in nove tesi: la storia umana - secondo la sua concezione teleologica - tende a due grandi mete, cioè costruire una società civile che faccia valere universalmente il diritto, e realizzare una federazione di popoli capace di porre fine alle controversie e di bandire le guerre.

\footnotetext{
${ }^{1}$ Il testo cui farò riferimento in queste pagine è tratto da I. KANT, Scritti politici e di filosofia della storia e del diritto, trad. di G. Solari e G. Vidari, a cura di N. Bobbio, L. Firpo e V. Mathieu, Torino, UTET, 1965, p. 123-139.

${ }^{2}$ «Una nota apparsa nella Gotaische gelehrte Zeitung ( 11 febbraio 1784) si esprimeva così: "Un'idea cara al professor Kant, è che lo scopo finale della specie umana è la realizzazione della costituzione politica più perfetta, ed egli augura che uno storico filosofo voglia ben intraprendere una storia dell'umanità concepita sotto questo punto di vista, che mostra fino a che punto l'umanità, nelle differenti epoche, si è allontanata o avvicinata a questo scopo, e che cosa c'è ancora da fare per raggiungerlo". Ė per rispondere all'aspettativa suscitata da questa nota che Kant scrisse nella Berlinische Monatsschrift del novembre 1784 l'articolo che divenne Idea...» da I. KAnT, La philosophie de l'histoire (opuscules), Paris, Editions Montaigne, 1947, p. 57.
} 
(BEDESCHI, 1994, p. 91). Nelle pagine che seguono sceglierò e commenterò alcune delle nove tesi del testo, iniziando dalla quarta.

"Il mezzo di cui la natura si serve per attuare lo sviluppo di tutte le sue [dell'uomo] disposizioni, è il loro antagonismo nella società, in quanto però tale antagonismo sia da ultimo la causa di un ordinamento civile della società stessa". L'antagonismo indica la socievole insocievolezza, la dimensione del "socievole" si riferisce alla "inclinazione" dell'uomo ad associarsi perché nello stato di società sente di poter sviluppare meglio le sue naturali disposizioni, mentre quella della insocievolezza rimanda alla "forte tendenza" a dissociarsi, perché l'uomo vuole rivolgere solo al proprio interesse ogni cosa e sa sia che gli altri resisteranno a ciò, sia che egli deve resistere a questa tendenza dell'altro. Per Kant la "dimensione negativa" è più utile, perché eccita tutte le energie dell'uomo, lo induce a vincere la sua tendenza alla pigrizia ed a conquistarsi un posto fra gli altri. ${ }^{3}$

Due costanti dell'antropologia kantiana: 1) l'uomo risulta istintivamente meno perfetto degli animali: questi ultimi diventano ben presto autosufficienti grazie al loro dinamismo istintivo, mentre l'uomo non presenta attitudini specifiche istintive e resta in balìa dei condizionamenti sociali, familiari e pedagogici, ecc.; 2) i rapporti interumani si basano molto di più sulla generalizzazione degli egoismi anziché sui sentimenti di simpatia. Superando l'opinione aristotelica circa l'uomo socievole per natura, Kant si sente più vicino ad Hobbes nel ritenere l'uomo insocievolmente socievole. E prosegue: la natura sfrutta la stessa insocievolezza dell'uomo (homo homini lupus!) per spingerlo a vivere in società. (MICCOLI, 1990, p.40). ${ }^{4}$

Questo ci permette di comprendere perché Kant non idealizzi né brami un ritorno allo stato di natura (positivamente inteso), qualificandosi al contrario come uno strenuo difensore della civiltà e della cultura. "Il conflitto con la natura, [... è] il presupposto, insieme al prodotto, della ragione, della legge morale e della civiltà. La ragione non è solo, come in Aristotele, condizione di felicità di "eudaimonia", ma anche rischio", (BODEL, 1981, p. 204-205).

Ancora di più però è importante notare che le passioni sono cause positive dell'entrata dell'uomo in società (al contrario della causa negativa costituita dalla mancanza di mezzi di sussistenza dell'uomo rispetto all'animale), perché per essere soddisfatte presuppongono l'alterità: la mia sete di onori può essere

\footnotetext{
${ }^{3}$ «Può sembrare che il passaggio dalla libertà sfrenata dello stato di natura (Kant è un giusnaturalista) alla libertà regolata della società civile, si configuri solo come qualcosa di necessario (per evitare i gravi inconvenienti della selvaggia libertà naturale), o solo come qualcosa di utile (poiché è soltanto nello stato civile che l'uomo realizza le condizioni del benessere e della sicurezza). In realtà non è così. [...] Kant, a differenza del precedente pensiero giusnaturalistico (si pensi a Locke), concepisce il passaggio dallo stato naturale allo stato civile non solo come qualcosa di necessario, o come qualcosa di utile o vantaggioso, ma anche come qualcosa di doveroso: ovvero tale passaggio costituisce per lui un dovere morale [...] perché è l'unico modo in cui gli uomini possono dominare e disciplinare i propri istinti e la propria naturalità (e quindi essere veramente uomini), possono garantirsi all'altrui prepotenza (ponendo fine al regno della mera forza), possono sviluppare e perfezionare le espressioni più alte e complesse della loro umanità» da G. Bedeschi, Il pensiero politico di Kant, Roma-Bari, Editori Laterza, 1994, p. 10-1 1.

${ }_{4}^{4}$ «La concezione di Kant è assai lontana «dalla concezione armonicistica della realtà socio-politica che caratterizza gran parte del pensiero tedesco settecentesco: realizzazione del diritto e affermazione della ragione sono piuttosto, per Kant, il risultato di un processo antagonistico che denuncia l'eco di una tradizione anglosassone - da Mandeville a Smith avvezza a vedere nella conflittualità sociale un fondamentale principio di sviluppo» (M. Mori, Illuminismo e criticismo nella filosofia della storia di Kant, in «Studi settecenteschi», 1988-89, VI, p.369)» da G. BEDESCHI, Il pensiero politico di Kant, Roma-Bari, Editori Laterza, 1994, p. 8.
} 
colmata solo da chi me li attribuisca; nell'ottica dei bisogni invece l'uomo vorrebbe restare da solo per avere tutto per sé. (PHILONENKO, 1986, p. 90).

\section{Kant ed il ruolo del popolo}

Tesi quinta: "il più grande problema alla cui soluzione la natura costringe la specie umana è di pervenire ad attuare una società civile che faccia valere universalmente il diritto". L'umanità può raggiungere lo sviluppo completo di tutte le sue facoltà solo in una società in cui ci siano tanto la massima libertà, e dunque il più grande antagonismo dei suoi membri, quanto la sicurezza più rigorosa dei limiti di tale libertà, affinché la mia libertà possa coesistere con quella degli altri. L'uomo deve accettare questo stato di co-azione perché deve sottrarsi ai mali che gli uomini si recano a vicenda, ed entrando nella costituzione civile si ha una regolamentazione della libertà del singolo, che perciò non può più danneggiare l'altro, e soprattutto gli impulsi umani si esprimono al massimo (metafora dell'albero che stretto fra gli altri, cresce dritto per cercare di arrivare di più al sole).

E necessario qui inserire una piccola parentesi: il problema di una strutturazione interna giusta per uno stato è fortemente collegato con la sua politica "estera". Se ne occupa la tesi settima: "il problema di instaurare una costituzione civile perfetta dipende dal problema di creare un rapporto esterno tra gli Stati regolato da leggi, e non si può risolvere il primo senza risolvere il secondo". Possiamo sottolineare nella tesi una divisione fra azione interna al singolo stato ed azione esterna che collega i vari singoli stati. Uno stato non può avere prima una costituzione perfetta e poi istituire un legame con altri stati. Perché gli stati creano una comunità fra di loro? Per lo stesso motivo per cui gli uomini si erano dati una costituzione unendosi in uno stato, ossia l'insocievolezza. La natura si serve della guerra, che richiede continuamente armamenti e porta miseria, nonché devastazioni, per fare arrivare gli stati alla conclusione - acquisita dolorosamente per esperienza, mentre la ragione avrebbe potuto presentarla subito - di entrare in una federazione di popoli in cui ogni stato possa sperare la propria sicurezza e la tutela dei propri diritti dalla stessa federazione dei popoli. Questo spiega anche quale peso possa avere avuto agli occhi di Kant la situazione travagliata che la Francia affrontava durante ed in seguito alla Rivoluzione Francese, che aveva scatenato un periodo di guerra fra la Repubblica francese e gli stati vicini, Prussia inclusa.

Tornando all'analisi della tesi quinta, Kant vi considera brevemente lo sviluppo realizzato fino ad arrivare al suo tempo e sottolinea: "noi siamo in alto grado colti sotto l'aspetto dell'arte e della scienza; noi siamo civili fino alla noia in tutto ciò che riguarda le forme e le convenzioni sociali. Ma per considerarci già moralmente progrediti ancora molto resta da fare». La nostra moralità fa parte ancora della cultura, cioè compiamo atti morali per seguire delle convenienze sociali ed il senso dell'onore; ma questo per Kant è incivilimento. L'unica soluzione a ciò è l'educarsi interiormente, cosa a cui i singoli cittadini si sforzano, ma a cui dovrebbero ovviamente contribuire anche gli stati invece di spendere in armamenti. 
La parola magica da cui l'epoca (dell'Illuminismo) si attendeva la soluzione di tutti i problemi sociali, etici ed economici, era educazione. $\mathrm{Si}$ voleva educare non solo il bambino ma anche il popolo [...]. In questa fede nella forza universale della pedagogia appare uno degli aspetti più caratteristici di questa nuova cultura, che era stata creata essenzialmente da insegnanti e da pastori [...]. La storia è il luogo dell'esercizio della libertà; la riflessione sulla storia è l'analisi etica della libertà umana. (MICCOLI, 1990, p. 29-40).

La filosofia della storia di Kant ha un intento pedagogico, che è quello di educare alla libertà. Tale primato che Kant assegna alla formazione dell'individuo e del cittadino sarà da riprendere più avanti sia in relazione al valore della pubblicazione di questo suo testo, sia in relazione alla modalità più significativa di fare avvenire dei cambiamenti all'interno degli stati.

Oltre alla possibilità di educazione, ogni individuo deve vedere rispettate le sue libertà civili. Le violazioni della libertà civile recano danno a tutto l'assetto interno dello stato, in quanto impedendo al cittadino di cercare il suo benessere con i mezzi per lui migliori si ostacola la sua alacrità al lavoro comune e perciò si sminuiscono le energie del tutto. Kant si riferisce in particolare modo al riconoscimento della libertà religiosa che produrrà per gradi l'illuminismo: l'uomo illuminato avrà una certa disposizione sentimentale verso il bene che si eleverà progressivamente fino ai troni arrivando ad influire sulle direttive fondamentali del governo. I reggitori prussiani non hanno riservato fondi per l'educazione ma avranno vantaggio se non ostacoleranno gli sforzi esercitati dal popolo in questo campo. Gli sforzi del popolo possono realmente portare a dei cambiamenti nella gestione della politica operata dai loro sovrani? E che tipo di sforzi? La domanda ci interessa in quanto esistono degli sforzi condotti con le armi, ossia i progetti rivoluzionari. Qui Kant accenna soltanto al campo dell'educazione e non ad un progetto rivoluzionario, come sarebbe stato quello intrapreso cinque anni dopo in Francia. Scrive in merito W. Euchner:

Se l'accademia non ha prestato tutta l'attenzione che i vari scritti di Kant sull'argomento meritavano, è forse perché li si prendeva per una volgarizzazione, che egli indirizzava ad un pubblico più vasto, a dei lettori di giornali. E Kant lo aveva fatto a questo scopo, per questo aveva scelto quel mezzo di diffusione. Per lui era centrale l'Aufklärung, l'educazione politica che poteva diffondere lo spirito repubblicano, la libertà di scrittura, che nessuno poteva ostacolare. [...] Perché la specie umana si sviluppi del tutto, serve: 1) costituzione repubblicana all'interno dello stato; 2) raggruppamento degli stati in una società delle nazioni. Ma la maggior parte dei governi al suo tempo erano in mano a principi autocrati: dunque i popoli come dovevano fare? L'imperativo morale impedisce di rovesciare i troni con le rivoluzioni, dunque si deve aspettare che l'illuminismo arrivi poco a poco ai principi. (EUCHNER, 1974, p. 17-23).

\section{Kant il rivoluzionario}

Kant era favorevole o contrario alla Rivoluzione Francese? È difficile rispondere con una posizione netta. Le interpretazioni critiche dei suoi testi in 
proposito sono alterne: a) Kant rivoluzionario, b) Kant reazionario, c) forti incongruenze presenti nei suoi testi e dunque un'evoluzione delle sue posizioni da favorevoli a contrarie, d) possibilità di dare una visione organica delle sue idee che manterrebbero dunque una linea sostanzialmente concorde con la rivoluzione.

Prendendo le mosse da quelle che sono le sue conclusioni circa il procedere del cambiamento della situazione sociale e politica, un procedere che dovrebbe andare dall'alto verso il basso, bisogna insistere sul fatto che Kant credeva fermamente nella possibilità di incidenza del popolo sulla condotta del sovrano. La forza di tale possibilità risiede nell'uso libero e pieno che ognuno può fare della propria ragione, uso che richiede già un notevole sforzo da parte del singolo, sempre tentato dalla via più comoda di seguire le direttive dei propri "tutori", invece di esercitare la propria capacità di pensiero discorsivo. Nell'età immediatamente anteriore a Kant, l'idea dello stato era quello dello stato paterno, arbitro della vita fisica e spirituale di ognuno. Ciò per Kant equivaleva alla morte dello spirito, alla servitù morale: nessuno può costringere un altro ad essere felice alla sua maniera. Un sovrano che fa così diviene un despota di fronte al quale il popolo diviene ribelle, (KANT, 1965, p. 171). Esercitando invece la libertà di poter fare un uso pubblico della propria ragione in ogni campo, il cambiamento politico seguirà senza sconvolgimenti armati. Uso pubblico della ragione è quello che fa un sapiente davanti all'insieme del pubblico che legge. Uso privato è quello che si fa della propria ragione quando si lavora al proprio posto in una istituzione pubblica, cioè nel caso in cui si debba solo obbedire, perché così si ha la garanzia che le istituzioni funzionino e si assicuri l'ordine pubblico. La discussione generale però su ciò che deve essere cambiato, offre una possibilità di cambiare la società, perché il re sentendo la maggioranza dell'opinione pubblica (visto che il prestigio legislativo del re sta nel fatto che incarna la volontà del popolo) (DENKER, 1974, p. 9-11), ${ }^{5}$ deciderà di agire secondo tale maggioranza.

Senza elencare nel dettaglio tutti i luoghi kantiani in cui si menziona la Rivoluzione, si possono prendere in considerazione alcuni passi dalla Critica del Giudizio (1790), da La Religione nei limiti della semplice ragione (1793), dai Principi metafisici della dottrina del diritto (1797) e da Il conflitto delle Facoltà (1798): 6

a) Nel $\S 65$ della Critica del Giudizio, Kant accenna, senza nominarla esplicitamente, alla Rivoluzione, non esprime un parere di stampo politico-sociale, ma apprezza l'uso del termine "organizzazione" per indicare la trasformazione statale operata dai Francesi. Questo termine merita attenzione in quanto una

\footnotetext{
${ }^{5}$ L'autore conclude da ciò che Kant fosse contrario alla rivoluzione francese, proprio in quanto i cambiamenti dovevano avvenire dall'alto.

${ }^{6}$ Cf. I. Kant, Critica del Giudizio, a cura di A. Bosi, Torino, UTET, 1993, p. 345-346 (nota a); cfr. Idem, La religione nei limiti della semplice ragione in Scritti morali, a cura di P. Chiodi, Torino, UTET, 1986, p. 518-519 (nota a); cfr. Idem, Il conflitto delle Facoltà, in Scritti politici e di filosofia della storia e del diritto, trad. di G. Solari e G. Vidari, a cura di N. Bobbio, L. Firpo e V. Mathieu, Torino, UTET, 1965, p. 214-230 e cfr. Idem, Principi metafisici della dottrina del diritto, p. 505-51 1 . Anche il testo del 1793 Sopra il detto comune: "Questo può essere giusto in teoria, ma non vale per la pratica" si espresse su queste tematiche. «Soprattutto interessò la seconda parte del saggio, in cui Kant per la prima volta esprimeva pubblicamente ed esplicitamente le sue idee politiche, e le esprimeva nel momento dei grandi avvenimenti della Francia rivoluzionaria, dei quali il saggio parve costituire il pratico commento» in Idem, Nota storica, 90. In proposito cfr. anche I. FETSCHER, "Emmanuel Kant et la Révolution française" in Emmanuel Kant 1724/1974. Kant comme penseur politique; Edité par Eduard Gerresheim, 1974 Inter Nationes Bonn - Bad Godsberg, p. 27-43.
} 
forma di governo in cui i cittadini sono e fini e mezzi gli uni per gli altri. L'idea kantiana di "organizzazione" va approfondita cogliendone il legame con "organismo" e "sistema". Se il primo di tali concetti trova esplicitamente spazio nella filosofia della religione (dagli stretti legami con l'antropologia e la filosofia della storia) kantiana, gli altri due sono fondamentali nella filosofia teoretica, nell'epistemologia kantiana, in quanto spiegano i "momenti ultimi" della strutturazione/costituzione della ragione umana che imposta tutta la formazione della conoscenza. Si può dire che lo stato si struttura in analogia alla ragione? Che entrambi sono organizzazioni/organismi (e dunque sistemi)? Il sistema, concetto tipico di Wolff, è l'opposto dell'aggregato: per spiegare ciò, Kant ricorre ad esempi tratti dalla chimica e dalla scienza della natura (non più dalla fisica). Parlando di "acqua pura" o "terra pura", Kant si riferisce ad enti che non esistono in natura, ma che indicano il desiderio di unità che opera all'interno della nostra dinamica conoscitiva guidata dalla ragione: si tratta dell'uso regolativo delle idee, necessario per dare il massimo di unità possibile alle conoscenze particolari, avvicinandosi così all'universalità. Possiamo chiederci se anche lo stato ricomponga così le libertà dei singoli cittadini, considerando in qualche modo che lo stato non serva a nulla se non ci sono i singoli cittadini liberi. Cosa significherebbe allora qui che si può solo approssimare la regola all'universalità? ${ }^{7}$

b) Ne La Religione nei limiti della semplice ragione, (KANT, 1986, p. 518-519), sempre in una nota, Kant commenta una delle obiezioni classiche rivolte ai movimenti rivoluzionari. Non si può essere d'accordo con chi sostiene che un popolo non è maturo per la libertà; "se fosse vero, sarebbe impossibile giungere alla libertà perché non si può diventare maturi per essa se prima non vi si è stati posti». I sovrani possono ritenere che il tempo di spezzare le catene del popolo sia in un'epoca ancora lontana, ma non possono sostenere in linea assoluta che la libertà non è adatta a quanti un tempo sono stati sottomessi; cioè è più comodo, ma non più giusto.

c) Fra l'altro, come Kant precisa ne Se il genere umano sia in costante progresso verso il meglio, ossia nella seconda parte de Il conflitto delle Facoltà, la presunta immaturità di un popolo per la libertà, non corrisponde alla natura umana, ma è il prodotto dell'ingiusta coazione dei despoti. Tale coazione rende i popoli proclivi ad insorgere. È questo un testo in cui si pone una esplicita domanda sul progresso. Per comprenderlo, ci si deve soffermare brevemente

\footnotetext{
${ }^{7}$ Se volessimo procedere oltre, potremmo avvalerci di alcune considerazioni dello studioso kantiano Silvestro Marcucci, il quale prende le mosse dal fatto che la base dell'unità sistematica della natura è costituita da tre "principi logici», ma con base trascendentale: il primo principio è il principio dell'omogeneità, il secondo (opposto al primo) è quello della specificazione; il terzo è quello della continuità delle forme, come una scala continua degli enti. Grazie a questi tre principi otteniamo l'«unità sistematica» delle nostre conoscenze della natura. Marcucci afferma così che: "La ragione spiana all'intelletto il suo campo con un principio dell'omogeneità del molteplice sotto generi superiori; con un principio della varietà dell'omogeneo sotto specie inferiori; e, per completare l'unità sistematica, essa aggiunge ancora una legge dell'affinità di tutti i concetti, la quale esige un passaggio continuo da ciascuna specie a ciascun'altra per mezzo d'un aumento graduale di differenza. [...] La prima legge vieta la dispersione della molteplicità dei diversi generi originari e raccomanda l'omogeneità; la seconda, invece, modera questa tendenza all'accordo, ed ordina una distinzione tra le sottospecie, prima che ci si rivolga con un concetto generale agli individui. La terza riunisce queste due, prescrivendo, insieme con la suprema molteplicità, anche l'omogeneità per un passaggio graduale da una specie all'altra; il che rinvia a una sorta di affinità tra i diversi rami, in quanto tutti insieme sono usciti da uno stesso tronco». S. MARCUCCI, Guida alla lettura della "Critica della Ragion Pura" di Kant, Roma-Bari, Editori Laterza, 1997, p. $117-1$ 18. Quello che noi possiamo chiederci è se queste tre leggi possano essere applicate, in un'ottica kantiana, anche alla costituzione di uno stato in cui si articoli un corretto rapporto fra istituzioni superiori, gruppi intermedi di appartenenza e singoli cittadini. E possibile qualche collegamento con i principi di sussidiarietà e di solidarietà che reggono da tempo l'etica sociale?
} 
sull'origine dello stesso, che si occupa del conflitto fra facoltà filosofica e quella teologica, fra quella filosofica e quella giuridica, fra quella filosofica e quella medica. Le altre facoltà tre erano considerate superiori rispetto a quella di filosofia vista come inferiore, ma il vero conflitto stava nel fatto che le facoltà pratiche erano indirizzate all'azione, alla conservazione dell'ordine costituito, mentre la filosofia era dominata solo dallo spirito critico senza considerare le conseguenze di ciò per l'ordine pratico. Il conflitto delle Facoltà in tutte le sue parti cade nel periodo di reazione allo spirito illuministico e rivoluzionario, che coincide col regno di Federico Guglielmo II (17 agosto 1786 - 16 novembre 1797). A causa della Rivoluzione Francese vennero represse quelle manifestazioni della libera speculazione critica che potevano sovvertire le basi tradizionali della condotta: perciò mentre la facoltà di filosofia era sospettata, quelle pratiche erano particolarmente favorite. L'adesione morale di Kant alla Rivoluzione Francese insospettì ancora di più. Dapprima Kant fu limitato dal punto di vista della critica all'ortodossia religiosa, poi venne la volta del conflitto con la facoltà di giurisprudenza che era asservita alla reazione. I giuristi e gli uomini di stato o ignoravano la Rivoluzione Francese o la combattevano come nemica. Kant non fu scoraggiato da questo atteggiamento pessimistico e scrisse la seconda parte del testo, censurato nel 1797 per l'apologia della Rivoluzione Francese che conteneva. La seconda parte, mentre vuole essere la valutazione della rivoluzione dal punto di vista del criticismo, è una nuova conferma dell'idea espressa da Kant fin dal 1784 nell'Idea di una storia universale, che il progresso dell'umanità verso il meglio è essenzialmente, nelle sue manifestazioni storiche, affermazione dell'idea di diritto, (KANT, 1965, p. 84-87).

Torniamo ora alla domanda sul progresso: perché porla? Perché durante l'epoca rivoluzionaria si erano prodotti innumerevoli crimini. Si poteva dunque parlare di un progresso continuo dell'umanità verso una moralità sempre più perfetta?

Di un avvenimento del nostro tempo che rivela questa tendenza morale della specie umana. [...] Trattasi solamente del modo di pensare degli spettatori che si rivela pubblicamente nel gioco delle grandi rivoluzioni e che manifesta una partecipazione universale e tuttavia disinteressata dei giocatori di un partito contro quelli dell'altro, malgrado il pericolo del danno che può ad essi derivare da tale spirito partigiano; ma (per la sua universalità) dimostra un carattere della specie umana in generale e ad un tempo (per il suo disinteresse) ne dimostra un carattere morale fondamentale, che fa non solo sperare nel progresso verso il meglio, ma costituisce già di per sé un tal progresso nella misura in cui esso può essere attualmente raggiunto. La rivoluzione di un popolo di ricca spiritualità, quale noi abbiamo veduto effettuarsi ai nostri giorni, può riuscire o fallire; essa può accumulare miseria e crudeltà tali che un uomo benpensante, se anche potesse sperare di intraprenderla con successo una seconda volta, non si indurrebbe a tentare a tal prezzo l'esperimento; questa rivoluzione, io dico, trova però negli spiriti di tutti gli spettatori (che non sono in questo gioco coinvolti) una partecipazione d'aspirazioni che rasenta l'entusiasmo, anche se la sua manifestazione non andava disgiunta da pericolo, e che 
per conseguenza non può avere altra causa che una disposizione morale della specie umana, (KANT, 1965, p. 218-219).

Questo testo merita una serie di considerazioni:

i) l'avvenimento che rivela la tendenza morale è un modo di pensare che appare pubblicamente (abbiamo visto prima l'insistenza kantiana sulla dimensione dell'esercizio pubblico della ragione), è possibile perciò che il pensiero sia un avvenimento di ordine morale? E quale tipo di pensiero lo sarebbe? Solo un pensiero che partecipa alla Rivoluzione Francese? Allora è la rivoluzione che ha qualche carattere morale da "trasmettere" ad un pensiero su se stessa? Oppure è la partecipazione del pensiero ad un qualsiasi evento storico a fare slittare un azione intellettuale sul piano della morale?

ii) La partecipazione deve essere universale: ma cosa significa universale? Sicuramente si trattava del pensiero di una parte dell'umanità (tanto è vero che c'erano posizioni reazionarie); dunque come fare il salto all'universalità? E deve essere anche disinteressata, il che implica che non porti nessun "guadagno" per il suo fautore. Cosa è allora questa partecipazione? Un bisogno, come il mangiare? Un dovere? Disinteressato potrebbe essere sinonimo di puro, cioè senza contaminazione di elementi di altra natura, qualcosa di scevro da elementi materiali? $O$ qualcosa di compiuto in sé?

iii) sono molto simili le caratteristiche di questa partecipazione con alcune di quelle del bello, che piace appunto universalmente, disinteressatamente, senza concetto. E perché Kant insiste tanto sui termini "gioco" e "spettatori", che rimandano alla dimensione estetica, al libero gioco delle facoltà di cui si parla nella Critica del Giudizio ed alla contemplazione tanto della natura, quanto dell'opera d'arte? Che rapporto c'è fra storia, arte e morale?

Noi apprendiamo gli eventi storici da un punto di vista estetico [un orribile massacro] e li giudichiamo ripugnanti o sublimi. E così i popoli marginalizzati, sul declino, ci ispirano un sentimento che non è solamente fondato sulla curiosità, ma anche sul dispiacere estetico di vedere dei popoli così lontani dalla nostra luce. Tra il fine ultimo, momento naturale, e l'Idea etica, il sentimento estetico funziona come mediatore, ben inteso che non potrebbe sostituirsi a nessuno dei due. Se questo sentimento estetico vuole valere da se stesso come principio, il rischio è il razzismo, così manifesto in Buffon p.e., per cui l'uomo marginalizzato non è più un prossimo ma un abbrutito. [In relazione al passo testé citato da Il conflitto delle facoltà] Qui il sentimento o il giudizio estetico funziona correttamente nel suo contenuto e nella sua mira. Dal punto di vista del contenuto Kant parla di spettatori che contemplano un gioco e che sono presi da entusiasmo e da simpatia (cosa che può essere pericolosa in un regime ostile alle idee rivoluzionarie). Dal punto di vista della mira si noterà che l'entusiasmo affonda le sue radici nella disposizione morale dell'umanità che mette in luce gli attori in quanto esseri storici. (PHILONENKO, 1986, p. 44-45). ${ }^{8}$

\footnotetext{
${ }_{8}$ «a Fichte a Kant, da Wieland a decine di altri scrittori e filosofi tedeschi meno noti, la rivoluzione francese è spesso definita uno "spettacolo": tremendo, sanguinoso, eppure capace di attrarre gli animi con forza irresistibile e di elevarli al di sopra della mediocrità quotidiana. Orrore e piacere vi si mescolano in proporzioni differenti e lo sgomento per la magnitudine di tale visione si accompagna alla speranza o al timore di radicali sconvolgimenti. Essa è tuttavia avvertita subito in Germania come un fenomeno di portata universale, un dramma nuovo e improvviso, i cui esiti ancora ignoti decideranno le sorti dell'umanità. Più che uno scenario di tragedia - in senso aristotelico, ossia quale intreccio purificatore di azioni che evocano "paura e pietà" -, la rivoluzione ha per sfondo il paesaggio del sublime. Un sublime politico, generato dalle
} 
iv) Tale partecipazione di aspirazioni rasenta l'entusiasmo. Di quale tipo di aspirazioni si parla qui? Del volere vivere una situazione analoga a quella della Rivoluzione? Kant specifica però che siamo nel piano del pensiero: dunque pensare una realtà è già viverla? Entusiasmarsi a qualcosa non è appassionarcisi? Le passioni rientrano allora nell'ambito morale grazie al giudizio storico?

v) Un evento storico concreto influisce sulla disposizione morale dell'uomo, la quale porta i singoli ad esprimere liberamente il loro pensiero. È questa dunque una prova che fra teoria e prassi non c'è poi distanza incolmabile? Questo esempio concreto che dà Kant, è sufficiente a mostrare che la sua visione della moralità include un recupero della dimensione storica, concreta, dei moventi reali e "passionali" nella determinazione dell'azione umana, in particolare modo di quell'azione che è l'esercizio del pubblico pensiero?

\section{Kant il reazionario}

Da quanto detto, sembrerebbe che Kant abbia sostanzialmente appoggiato la Rivoluzione francese, come un evento i cui frutti non erano stati solo e tanto a vantaggio della Francia stessa, quanto portatori indiretti di un progresso a livello di umanità tutta.

Nel 1797 però, Kant aveva pubblicato i Principi metafisici della dottrina del diritto (KANT, 1965, p. 505-511), in cui si trova una critica severa agli eccessi della Rivoluzione: (AA.VV., 1989, p. 110), ${ }^{9}$ viene condannata ogni azione rivoluzionaria ed anche lo stesso diritto di resistenza all'ingiustizia. Soprattutto su questo testo si basano gli autori che interpretano le posizioni kantiane come reazionarie. Sono tuttavia possibili delle vie di mezzo: Kant ammirava nella Rivoluzione il manifestarsi di quelle forze cieche, naturali, spontanee, che nella storia agiscono indipendentemente da ogni prevenzione umana per il trionfo di ciò che è vero e giusto, ma non vi credeva realizzata la sua concezione del diritto e dello stato. Lo stato giuridico kantiano è liberale nel senso che esso vuole rispettata il più possibile la libertà esterna nell'individuo e la vuole solo regolata e coartata nei limiti imposti non da considerazioni utilitarie, eudemonistiche, ma da un principio di ragione capace di originare almeno esteriormente una volontà e azione comune.

azioni degli uomini, ma del tutto analogo al "sublime dinamico" innescato nella coscienza dall'attività spontanea della natura. [...] La tremenda maestà dell'evento, il suo mostrare, in un dissidio irrisolto, la trasparenza in forma sensibile di "idee" che alludono ad una destinazione più alta dell'uomo rispetto alle potenze scatenate dalla natura, rendono gli individui partecipi - coinvolti a distanza di sicurezza - di un dramma che è indissolubilmente naturale e morale. Le forze selvagge del mondo esterno che sembrano voler distruggere l'uomo e annullarne l'importanza, finiscono così per restituirgli la consapevolezza della sua superiorità morale», R. Bodei, "Le Dissonanze del Mondo. Rivoluzione Francese e Filosofia Tedesca tra Kant e Hegel" in AA.VV., L'Eredità della Rivoluzione Francese, a cura di François Furet, Bari, Editori Laterza, 1989, p. 103-104.

9 «E i Tedeschi - almeno per qualche tempo - osservano, senza esserne coinvolti, gli eventi che si sviluppano, geograficamente, appena oltre il confine. Salutano allora la rivoluzione come un sole luminoso, una giornata campale nella lunga storia dell'umanità. Ma quando essa cambia volto (perché appare in pericolo ai suoi stessi protagonisti e perché ad essa si intrecciano la guerra civile e quella esterna), molti scrittori che l'avevano accolta con convinto e sincero entusiasmo le voltano le spalle inorriditi. [...] inquietanti paradossi seguono dalla teoria e dalla pratica giacobina secondo cui, per sopravvivere, la repubblica ha bisogno del dispotismo, la «virtù» del Terrore e la ragione stessa della violenza. [...] Bisogna guidare gli uomini alla libertà, all'eguaglianza e alla fraternità mediante la ragione, ma, se resistono, mediante il terrore e la violenza. La ragione e la virtù disarmate non sono sufficienti a spezzare le lunghe "catene della schiavitù» e dell'egoismo, a far governare quell'entità morale immaginaria che è il popolo, nel cui nome i giacobini parlano», R. Bodei, "Le Dissonanze del Mondo. Rivoluzione Francese e Filosofia Tedesca tra Kant e Hegel." 
Alcuni vollero fare di Kant il filosofo della borghesia, trionfante proprio in quegli anni della rivoluzione francese. Si può anzi rilevare nella borghesia da un lato l'avversione, che fu propria di Kant, contro la concezione democratica ed egualitaria della libertà, che porta alla tirannide popolare; dall'altro la preferenza, che fu anche di Kant, verso costituzioni politiche che rispettano la ricca, varia esplicazione dell'individualità empirica e traggono dalla natura stessa della libertà la necessità di un ordine giuridico che ne impedisce con misura razionale e uniforme le intemperanze. ${ }^{10}$

È evidente l'incompatibilità presente fra i testi analizzati sinora e quest'ultimo, ma fino a che punto arriva? Per rispondere, dobbiamo cercare di analizzare due ordini di questioni: per quale motivo Kant rifiuta il diritto alla rivoluzione? $\mathrm{E}$ come si caratterizzano gli atti rivoluzionari?

Iniziamo dalla prima domanda. I motivi kantiani sono sostanzialmente due e sarebbero forse condivisibili anche da un governo andato al potere grazie ad una rivoluzione; il primo motivo ci porta a considerare che:

\begin{abstract}
non si può inserire in nessun ordinamento giuridico una norma che preveda esplicitamente il rovesciamento della Costituzione (questo sì sarebbe un "suicidio» per qualsiasi Stato). Quando ha luogo una rivoluzione, ossia il "rovesciamento violento di una Costituzione difettosa", ciò accade sempre, per Kant, al di fuori del diritto, e in particolare del diritto vigente. Nel sostenere tale teoria, egli non si allontana, del resto, da una dottrina politica consolidata, secondo cui il diritto medesimo nasce dalla violenza, (SCHRECKER, 1939, p. 394-425).
\end{abstract}

Se si scatena un conflitto fra il popolo ed il sovrano, il popolo non può presentarsi come giudice nella propria causa, e se nomina un giudice esterno, un arbitro supremo, correrà di nuovo il rischio che costui diventi a sua volta un nuovo sovrano/tiranno. L'unica soluzione può dunque venire dall'alto: deve essere il sovrano stesso a promuovere una riforma (SCHRECKER, 1939, p. 394-425). Il secondo motivo è legato al fatto che nel momento storico preciso in cui Kant scrive, si svolgeva la rivolta in Vandea contro le politiche imposte dal governo giacobino da Parigi: giustificare il diritto di resistenza, avrebbe significato giustificare la reversibilità dell'assetto politico stabilito dalla rivoluzione, assumere una posizione nettamente anti-giacobina.

In questa discussione rientra anche la considerazione kantiana della convocazione degli Stati Generali da parte del re Luigi XVI per il $1^{\circ}$ maggio 1789. Per Kant, con questa convocazione, il re - senza saperlo - aveva dato l'avvio ad una riforma (motivo per cui gli eventi seguenti non sarebbero stati una rivoluzione): conferendo loro il potere legislativo per ridefinire il problema delle tasse, il re si sarebbe dimesso dalla propria sovranità, passandola al popolo.

Per quanto riguarda il valore degli atti rivoluzionari, c'è da compiere una distinzione rispetto ai piani su cui valutarli. Gli atti rivoluzionari sono giuridicamente criminali e dunque immorali, ma ciò non si oppone al fatto che lo stato di cose dopo la Rivoluzione possa essere dal punto di vista della ragione e della giustizia, superiore al regime prerivoluzionario; il che implica dunque che per Kant non

\footnotetext{
${ }^{10}$ Cf. per esempio I. Kant, Scritti politici e di filosofia della storia e del diritto, tradotti da G. Solari e G. Vidari, Torino, UTET,
} 1965 [Introduzione di Gioele Solari, p. 19-23] 
siano giustificabili gli sforzi della Restaurazione. Non si giudica della perfezione di una cosa nel suo dominio, cioè del grado in cui questa cosa realizza ciò che deve essere, partendo dalla sua storia o dalla perfezione morale dell'azione che l'ha generata, azione che è responsabile davanti ai giudici e secondo delle leggi differenti da quelle che sono chiamate a giudicare la cosa creata. La giurisprudenza e la morale giudicano deli atti scaturiti dalla volontà dei singoli, la filosofia della storia si interessa dei gradi di perfezione delle condizioni successive dell'umanità, (SCHRECKER, 1939, p. 403-404).

L'umanità ha attraversato epoche e condizioni di vita diverse: la filosofia della storia domanda quale siano state più perfette. Possiamo chiederci allora: quale rapporto fra la storia e la morale? Si può giudicare un'epoca? E, se sì, in base a cosa? O - ad un livello più basso - se si possono giudicare solo azioni i cui autori e responsabili siano ben individuabili, come si fa a dare un giudizio su un evento che ha svariate cause determinanti? Domande del genere costituiscono veramente il cuore di ogni riflessione sullo statuto della filosofia della storia. Distinguendo fra giurisprudenza (giudice) e storia/filosofia della storia (storico/filosofo della storia), Kant sembra anticipare questioni di metodologia storica, che reimposteranno questa disciplina a partire dal '900.'1 Anche l'occuparsi dei gradi di perfezione delle varie epoche storiche implica che, per quanto lo storico non emetta giudizi, egli abbia almeno una tavola di valori in base a cui stabilire per rapporto di convenienza, la maggiore o minore perfezione degli oggetti da lui presi in considerazione. Come stabilire una tale gerarchia di valori per i periodi storici? Quale valore/bene/fine deve essere raggiunto in una determinata epoca? Quale senso ha la domanda sulla finalità in filosofia della storia? Come ha risposto Kant a queste domande?

Relativamente all'elaborazione di una filosofia della storia, Kant conclude il suo saggio con quanto segue. L'idea di ciò che dovrebbe essere il corso del mondo umano se esso dovesse adeguarsi a certi fini razionali, ci è utile per redigere una storia: essa suppone che la natura proceda secondo un disegno verso uno scopo anche nel gioco della libertà umana. L'utilità di questa idea sta nel suo lavorare come filo conduttore per rappresentarci almeno nell'insieme come un sistema quello che altrimenti ci apparirebbe come un informe aggregato di azioni umane. Si deve cominciare dalla storia greca, passando per la romana e per la sua distruzione ad opera dei barbari, per arrivare fino a noi. Questa storia può chiarire il gioco delle cose umane; presagire i futuri mutamenti politici degli stati; aprire una visione confortante dell'avvenire.

Bisogna scegliere un particolare punto di vista nella concezione dell'universo: Kant ne sta scegliendo uno in cui la natura (o meglio la Provvidenza) dà una

\footnotetext{
${ }^{11}$ Cf. il problema dell'imparzialità in M. Bloch, Apologia della storia o Mestiere di storico, Torino, Einaudi, 1998, p. 104-105. Ci sono due modi di essere imparziali, quello dello studioso e quello del giudice. In comune hanno l'onesta sottomissione alla verità. Lo studioso provoca le esperienze che confuteranno la sua teoria, il giudice - per quanto il suo cuore abbia già votato - interroga i testimoni con la preoccupazione di conoscere i fatti come sono accaduti. Ė in entrambi i casi un obbligo di coscienza che non può essere discusso. Ad un certo punto le loro strade divergono: lo studioso osserva spiega e si ferma; il giudice deve anche emettere la sentenza. Se lo fa secondo la legge senza simpatie personali, è imparziale, dal punto di vista dei giudici ma non da quello degli studiosi. Si condanna o si assolve un uomo solo schierandosi per una tavola di valori, che nessuna scienza positiva potrà dedurre. Che un uomo ne abbia ucciso un altro è un fatto suscettibile di prova, ma punirlo presuppone che lo si consideri colpevole, il che non è un'opinione su cui tutte le civilizzazioni abbiano avuto convergenza. Per lungo tempo lo storico è stato considerato come una specie di giudice degli inferi, che distribuiva elogi o biasimi ai morti.
} 
giustificazione allo sviluppo storico dell'uomo, senza bisogno di dovere cercare in un altro mondo un disegno perfetto e razionale. Questa storia filosofica - che non si sostituisce ad una redazione puramente empirica della storia - ha anche il piccolo vantaggio di fornire ai capi di stato un modo per trasmettere ai posteri un buon ricordo di sé, in base a quanto hanno favorito lo sviluppo cosmopolitico. ${ }^{12}$

Sulla filosofia della storia kantiana, la bibliografia è assai ampia: è molto difficile offrire una visione complessiva ed unitaria anche di quest'ultima. Riportiamo solo alcune suggestioni, che si ricollegano alla considerazione delle passioni in quanto motore della storia e che potrebbero aprire anche ad un confronto con Hegel su tali tematiche. Secondo l'interpretazione di Remo Bodei, Kant ed Hegel focalizzano soprattutto:

l'idea della eterogenesi dei fini, ossia della involontaria metamorfosi che gli scopi dei singoli uomini subiscono quando le loro azioni, confluendo in quelle di tutti, travalicano le intenzioni individuali. Kant si inserisce così, in maniera originale, nella tradizione moderna di pensiero, inaugurata dalla Favola delle api di Mandeville, per la quale non solo i vizi privati danno luogo a pubbliche virtù, ma sono proprio tali vizi a fungere da molle della civiltà. È infatti dalla ricerca di guadagno e dall'avarizia che nasce il commercio e, quindi, il benefico scambio tra gli uomini [...]. È quindi una caratteristica del modello kantiano - e di tutte le filosofie della storia che si pongono sul suo solco - la volontà di recuperare a posteriori quel senso che gli uomini hanno erogato in precedenza senza rendersene completamente conto. Tale senso complessivo, inoltre, non è più indagato come il prodotto di cause finali, individuando cioè gli scopi che gli uomini si sono prefissi. Si cerca piuttosto di comprenderlo interrogando una logica anonima della storia, diventata autonoma e indipendente dalle motivazioni individuali delle azioni. [...] Leggi, istituzioni, strutture collettive sono il risultato materializzatosi dell'operare di miliardi di nostri simili, vissuti in tempi e in luoghi differenti. Abitiamo in un mondo che è stato reso sensato dagli uomini e dobbiamo inserirci in questo mondo, comprendendolo e riproducendolo incessantemente, (BODEL, 1997, p. 48-49).

Tale visione della storia ha una chiara ricaduta anche sulla concezione di "storiografia" del nostro autore, che diviene una elaborazione di idee regolative:

La storiografia diventa quindi una grande opera di decifrazione di noi stessi, uno strumento per dar significato alla nostra vita. Se la storia non accetta più modelli teologici, se aspira alla spiegazione degli eventi attraverso altri eventi, per linee interne, bisogna allora capire qual è il motore della storia. Per Kant si chiama concordia discors. Vale a dire: la storia procede in avanti perché vi è una benefica competizione fra individui che hanno bisogno l'uno dell'altro. [...] si possono tuttavia elaborare solo "idee regolative", capaci di fungere da "filo conduttore" nella comprensione di eventi altrimenti irrelati, (BODEL, 1997, p. 49-50). ${ }^{13}$

\footnotetext{
12 Questo è quanto si può desumere dalla conclusione del suo testo Idea di una storia universale dal punto di vista cosmopolitico [1784], in I. Kant, Scritti politici e di filosofia della storia e del diritto, trad. di G. Solari e G. Vidari, a cura di N. Bobbio, L. Firpo e V. Mathieu, Torino, UTET, 1965, p. 138-139.

${ }^{13}$ "La sua filosofia della storia è un modo di guardare alla storia che è accettabile per la mente umana sia nella sua dimensione teoretica che in quella pratica. La sua visione teleologica soddisfa sia la domanda della ragione teoretica che il mondo sia visto come un tutto coerente inerentemente, sia la domanda della ragione pratica che il mondo sia visto come compatibile con l'esistenza possibile della libertà umana», H. WiLliams, Kant's Poltical Philosophy, Oxford, Basil Blackwell, 1983, p. 20.
} 
Continuando nell'analisi di alcuni passi dai Principi metafisici della dottrina del diritto, si possono evidenziare anche varie somiglianze fra le proposte della filosofia kantiana e la Dichiarazione dei Diritti dell'uomo e del cittadino emanata il 26 agosto 1789 dall'Assemblea nazionale. Essa affermava come diritti naturali e imprescrittibili dell'uomo la libertà, la proprietà, la sicurezza e la resistenza all'oppressione. Si sancivano i principi della sovranità nazionale e della divisione dei poteri, la libertà di pensiero, di parola e di stampa, l'eguaglianza di fronte alla legge. In Francia non si aveva una conoscenza della filosofia kantiana così completa come quella che la filosofia tedesca aveva degli avvenimenti che sconvolgevano il mondo. Ciononostante, le somiglianze di cui sopra, possono essere facilmente comprese, pur senza ricorrere ad un diretto influsso di una delle due parti sull'altra, tenendo conto che la Dichiarazione dei diritti dell'uomo e del cittadino (e la legislazione che ne segue) ed alcune dottrine kantiane avevano in gran parte fonti comuni: il diritto di natura degli umanisti, il radicalismo inglese, il costituzionalismo americano, Montesquieu, Rousseau, (SCHRECKER, 1939, p. 395-396).

Va inoltre considerato che la costituzione repubblicana soltanto poteva garantire i diritti innati, inalienabili. Essa è definita dal modo in cui lo stato esercita il suo potere, che può essere repubblicano se ci sono due principi fondamentali, precisamente quelli cui la Rivoluzione Francese aveva dato attuazione, ossia il sistema rappresentativo e la separazione dei poteri, (SCHRECKER, 1939, p. 399-400). Per Kant poi, che segue in questo la teoria dei diritti naturali ed imprescrittibili dell'uomo, questi diritti emanano direttamente ed immediatamente dalla ragione e non sono riducibili a nessun altro dato. Non è né l'uomo né la società che li dispensa; la società al contrario li suppone come elementi costitutivi (ciò in cui si distingue da una banda organizzata), ed essa assicura loro solo l'esecuzione. Così questi diritti costituiscono, per così dire, l'a priori del dominio sociale, proprio come le forme dell'intuizione pura e le categorie sono gli elementi costituenti della conoscenza: essendo gli uni e gli altri le condizioni necessarie e trascendentali della possibilità del lavoro razionale sul loro terreno rispettivo.(SCHRECKER, 1939, p. 417).

a) Condanna della grande proprietà fondiaria della nobiltà e della Chiesa: Kant condanna la proprietà acquisita solo a causa dei privilegi che favoriscono questi ordini. Per Kant è invece fondamentale la proprietà di un bene (un commercio, un negozio, un piccolo appezzamento terriero), il quale permetta l'indipendenza, l'autonomia del singolo cittadino rispetto ai suoi concittadini. Dalla coesione di questi piccoli proprietari = cittadini, nasce lo stato, (FETSCHER, 1974, p. 32-40).

b) Abolizione dei privilegi della nobiltà, che costituiva un ceto intermedio fra il sovrano assoluto ed il terzo stato, un ceto dai privilegi ereditari che comunque si caratterizzava come superiore al popolo stesso. Un uomo non può rifiutare la propria libertà ed il proprio dovere di decidere per sé; non può perciò essere fondato né dal sovrano, né dal popolo uno stato superiore al popolo stesso e che decida per lui. Come può allora il sovrano ottenere il risultato di emanare delle leggi che valgano per tutti, come se emanate da una repubblica? Ciò è possibile se si circonda di consiglieri leali e lungimiranti, ma soprattutto se adotterà come criterio per l'elaborazione di una legge, quello di domandarsi come avrebbe fatto il popolo se avesse dovuto prescriversi da se stesso una legge (il che equivale a 
riprendere la formula dell'imperativo categorico, di seguire una massima che possa essere elevata a legislazione universale). A ciò si aggiunge il fatto che il sovrano - come i suoi consiglieri - non può essere infallibile, in quanto uomo: dunque il popolo ha il diritto di esprimersi secondo la libertà di penna nei limiti del rispetto per la costituzione. In accordo con il programma del 1789 Kant afferma che uno dei diritti più preziosi dell'uomo è la libera comunicazione dei pensieri e delle opinioni. Condizione indispensabile della pace perpetua è che la costituzione accordi ai filosofi la libertà di parlare pubblicamente sugli affari dello stato.

c) Abolizione della servitù: nessuno può legarsi ad un altro in modo tale da cessare di essere persona. Fra l'altro se si può avere un contratto solo fra due persone private, nel momento in cui una delle due accettasse di cessare di essere persona il contratto non varrebbe, (FETSCHER, 1974, p. 39-42; KANT, 1965, p. 518-519).

Resterebbe da affrontare il problema della compatibilità fra quanto appena affermato e l'esistenza di un governo monarchico come era quello prussiano; Kant presenta più volte delle note a margine, nei testi che abbiamo preso in considerazione, in cui spiega che un popolo che abbia costituzione monarchica non è automaticamente autorizzato, dalle riflessione di Kant stesso, a nutrire il desiderio di rovesciare tale costituzione (cfr. in proposito i passi citati da Il conflitto delle Facoltà, nelle pagine precedenti - nota 15).

Da una parte abbiamo segnalato come la prospettiva kantiana non sia di per sé favorevole alla rivoluzione, ma piuttosto ad una sorta di evoluzione che provenga dall'alto; dall'altra restano le dissonanze nelle interpretazioni per cui alcuni vedono Kant come un vero e proprio reazionario, mentre altri non ritengono essenziale queste note a margine in difesa della monarchia prussiana. Di fatto alcuni anni dopo la morte di Kant, in Prussia furono attuate varie riforme (seppure limitate) portate avanti dalla volontà di cambiamento della fascia di alti funzionari che circondavano il re, e non dal popolo. Proprio da questo punto di vista "popolare",

la Germania non era affatto un luogo di delizie e di ordine. I contadini soffrivano spesso fame ed oppressione (soprattutto in Sassonia e in Slesia, dove vi furono infatti violenti moti popolari tra il 1790 e il 1792). Colonia e Berlino e altri centri minori erano invasi da mendicanti e vagabondi. Lo sviluppo delle manifatture e la loro stessa crisi producevano abbrutimento ed incrementavano la disgregazione sociale. [...] Se ci fu, nella storia della Germania, un periodo in cui la cultura tedesca lottò per far coincidere teoria e prassi (una separazione concettuale che è, per inciso, post-hegeliana), presente filosofico e presente storico, per adeguare le condizioni della Germania a quelle del mondo politico uscito dalla Rivoluzione Francese, fu appunto quello che abbiamo considerato [da Kant ad Hegel]. Il 'pensare la rivoluzione' mediante categoria filosofiche non fu allora separato - in diversi Tedeschi - dal tentativo di 'fare la rivoluzione', (BODEL, 1989, p. 126-127).

\section{Conclusioni}

Il sorgere della storia viene individuato da Kant nella realtà della "socievole insocievolezza" che obbliga l'uomo ad unirsi in una società civile con delle regole: l'uomo infatti ha una inclinazione ad unirsi con altri e sotto delle regole 
perché solo in società può sviluppare le sue disposizioni naturali, ma vorrebbe anche avere ogni cosa per sé (motivo per cui non desidera stare in società e rispettarne le regole). Le passioni ricoprono un ruolo importante nell'entrata in società e dunque nella realizzazione della storia: per soddisfare una passione "serve" infatti l'altro.

L'umanità può raggiungere lo sviluppo completo di tutte le sue facoltà solo in una società in cui ci sia la massima libertà per ognuno e la massina sicurezza per tutti (altrimenti le diverse libertà non potrebbero coesistere). Ora, l'educazione della libertà è l'obiettivo fondamentale della filosofia della storia kantiana, che ha dunque una natura teleologica. Nell'epoca in cui Kant scriveva però, i sovrani non riservavano fondi per l'educazione: doveva essere il popolo con i suoi sforzi ad occuparsi della propria auto-educazione.

Con l'entrata in gioco del popolo però, viene tirata in ballo anche la rivoluzione: esistono sforzi condotti con le armi come le rivoluzioni. Ora, la posizione kantiana nei confronti della rivoluzione, particolarmente quella francese, non è univoca. Sicuramente era centrale per lui l'attenzione all'educazione politica che poteva fare espandere lo spirito repubblicano, ma non potendosi rovesciare i troni con le rivoluzioni, sembrava che l'unica strada percorribile restasse quella dei principi illuminati.

Kant credeva fermamente nella possibilità di incidenza del popolo sulla condotta del sovrano: ciò era possibile per lui perché ogni uomo può fare un uso libero e pieno della propria ragione. Ora, visto che il re trae il suo prestigio dal fatto di incarnare la volontà popolare, ascoltando la maggioranza dell'opinione pubblica, il re ascolta il popolo e dunque quest'ultimo ha reale possibilità di incidere sul sovrano.

I passi kantiani presi qui in considerazione sono stati tratti dalla Critica del Giudizio (1790), da La Religione nei limiti della semplice ragione (1793), dai Principi metafisici della dottrina del diritto (1797) e da Il conflitto delle Facoltà (1798): nella Critica del Giudizio, Kant accenna alla Rivoluzione, apprezzando l'uso del termine "organizzazione" per indicare la trasformazione statale operata dai Francesi. Questo termine merita attenzione in quanto può essere avvicinato a quello di organismo e di sistema e permetterebbe di vedere nella organizzazione statale un movimento parallelo a quello della ragione che, nella sua ricerca della massima unificazione possibile delle conoscenza, lavora come un sistema.

$\mathrm{Ne}$ Se il genere umano sia in costante progresso verso il meglio, seconda parte de Il conflitto delle Facoltà, Kant si sofferma invece sulla giustificazione implausibile della presunta immaturità di un popolo per la libertà, come motivo per non concedergliela. Essa è infatti il prodotto dell'ingiusta coazione dei despoti, la quale spinge i popoli ad insorgere. Le insurrezioni però richiedono tutta una seria di atti criminosi che sembrano contraddire l'idea di un progresso continuo dell'umanità verso una moralità sempre più perfetta. L'importanza di questo passo sta - fra l'altro - nelle domande che ci genera sulla natura del pensiero: il pensiero può essere un atto morale? E tale moralità dipende dall'oggetto del pensiero (in questo caso la Rivoluzione Francese)? La partecipazione mediante il pensiero, all'evento della Rivoluzione Francese, ha delle caratteristiche molto simili a quelle del bello (universalità, disinteresse, mancanza di concetto): che rapporto c'è allora fra storia, arte e morale? 
La partecipazione alle aspirazioni del popolo francese rasenta inoltre l'entusiasmo: riguarda cioè il campo delle passioni che rientrano così in gioco, dopo essere state lette come motore della storia. Gli eventi storici di fronte a cui l'uomo si pone sembrano inoltre colmare la distanza fra teoria e prassi: l'esercizio del pubblico pensiero è infatti un atto che ingloba passione e moralità.

Dalla considerazione del testo del 1797 Principi metafisici della dottrina del diritto, è emersa al contrario la severa critica severa agli eccessi della Rivoluzione: viene condannata ogni azione rivoluzionaria ed anche lo stesso diritto di resistenza all'ingiustizia. Kant inoltre deplora dal punto di vista morale e legale gli atti rivoluzionari, ma non da quello della filosofia della storia che riconosce la perfezione maggiore della condizione stabilita dalla Rivoluzione rispetto a quella prerivoluzionaria.

Nel tirare le somme di quanto scritto finora, dobbiamo rilevare che da qui nascono due serie di domande: che rapporto c'è fra storia e morale? E come si può stabilire una gerarchia fra diversi periodi storici? Questo implicherebbe che ogni epoca cerchi di raggiungere un fine e che questi fini siano confrontabili. Quale senso ha la domanda sulla finalità in filosofia della storia? Come nel funzionamento della ragione umana che cerca l'unità massima possibile della conoscenza, così l'idea di un fine della storia offre un'occasione di unità, facendoci vedere le cose umane come un sistema e non come un mero aggregato. Questa storia filosofica può chiarire il gioco delle cose umane; presagire i futuri mutamenti politici degli stati; aprire una visione confortante dell'avvenire. La storia ha una sua logica autonoma ed indipendente dalle motivazioni individuali delle azioni, ma questa logica - questo senso - si comprendono solo a posteriori e nella rilettura dei meri eventi.

Ricordiamo poi che per Kant solo la costituzione repubblicana può garantire i diritti innati, inalienabili, ma affinché ci sia tale tipo di costituzione, servono il sistema rappresentativo e la separazione dei poteri, come la Rivoluzione Francese aveva adottato. Il popolo inoltre ha il diritto di esprimersi secondo la libertà di penna nei limiti del rispetto per la costituzione, proprio per andare a colmare le lacune dell'azione del re e dei suoi consiglieri. Kant segue così in qualche modo il programma del 1789, affermando che uno dei diritti più preziosi dell'uomo è la libera comunicazione dei pensieri e delle opinioni.

Da quanto detto, appare plausibile considerare - per quanto permangano le contraddizioni fra i due ordini di testi rispetto alla valutazione della Rivoluzione Francese - una certa omogeneità nel pensiero di Kant, in quanto egli riconosce in ogni caso una condivisione profonda di alcuni principi fondamentali della Rivoluzione del 1789.

Quando si tratta di filosofia, il termine "liberale", "conservatore", "reazionario" non hanno alcun senso preciso e possono riceverlo solo dalla ricerca filosofica stessa, una volta che (e nella misura in cui) essa avrà dato una definizione del progresso e fissato un orientamento alla storia. (E. WEIL, Hegel et l'État, Paris, Librairie philosophique J. VRIN, 1950, p. 23). 


\section{Riferimenti}

BEDESCHI, G. Il pensiero politico di Kant. Roma-Bari: Editori Laterza, 1994.

BLOCH, M. Apologia della storia o Mestiere di storico. Torino: Einaudi, 1998.

BODEI, R. "Le Dissonanze del Mondo. Rivoluzione Francese e Filosofia Tedesca tra Kant e Hegel" In: AA.VV., L'Eredità della Rivoluzione Francese, a cura di François Furet. Bari: Editori Laterza, 1989.

. Se la Storia ha un Senso. Bergamo: Moretti \&Vitali editori, 1997.

"Tenerezza per le cose del mondo: sublime, sproporzione e contraddizione

in Kant e in Hegel" In: AA.VV., Hegel interprete di Kant, a cura di Valerio Verra. Napoli: Prismi, 1981.

DENKER. R. "La théorie kantienne de la triple voie vers la paix mondiale ou: les intensions de la nature dans l'histoire" In: Emmanuel Kant 1724/1974. Kant comme penseur politique; Edité par Eduard Gerresheim, 1974 Inter Nationes Bonn - Bad Godsberg.

EUCHNER, W. "Kant philosophe du progrès politique" In: Emmanuel Kant 1724/1974. Kant comme penseur politique; Edité par Eduard Gerresheim, 1974 Inter Nationes Bonn - Bad Godsberg.

FETSCHER, W. "Emmanuel Kant et la Révolution française" In: Emmanuel Kant 1724/1974. Kant comme penseur politique; Edité par Eduard Gerresheim, 1974 Inter Nationes Bonn - Bad Godsberg.

KANT, I. Critica del Giudizio, a cura di A. Bosi. Torino: UTET, 1993.

. Critica della Ragion Pura. Bari: Editori Laterza, 1993.

- Idea di una storia universale dal punto di vista cosmopolitico [1784] in Scritti politici e di filosofia della storia e del diritto, trad. di G. Solari e G. Vidari, a cura di N. Bobbio, L. Firpo e V. Mathieu. Torino: UTET, 1965.

- Il conflitto delle Facoltà, in Scritti politici e di filosofia della storia e del diritto, trad. di G. Solari e G. Vidari, a cura di N. Bobbio, L. Firpo e V. Mathieu. Torino: UTET, 1965.

. La religione nei limiti della semplice ragione in Scritti morali, a cura di P. Chiodi. Torino: UTET, 1986.

. La philosophie de l'histoire (opuscules). Paris : Editions Montaigne, 1947.

. Principi metafisici della dottrina del diritto, in Scritti politici e di filosofia della storia e del diritto, trad. di G. Solari e G. Vidari, a cura di N. Bobbio, L. Firpo e V. Mathieu. Torino: UTET, 1965.

MARCUCCI, S. Guida alla lettura della "Critica della Ragion Pura" di Kant. RomaBari: Editori Laterza, 1997.

MICCOLI. P. Ragione e storia. Da Kant a Sartre. Roma: Borla, 1990.

PHILONENKO, A. La théorie kantienne de l'histoire. Paris: Librairie Philosophique J. Vrin, 1986. 
SCHRECKER, P. "Kant et la Révolution française" In: Revue philosophique de la France et de l'Étranger (1939), p. 394-425.

WEIL, E. Hegel et l'État. Paris: Librairie philosophique J. Vrin, 1950.

WILLIAMS, H. Kant's Poltical Philosophy. Oxford: Basil Blackwell, 1983.

\section{Sobre a autora}

Sara Bianchini

Doutora em Filosofia. Professora da Universidade Gregoriana, Roma, Itália.

Email: bianchini@unigre.it

Recebido em 13/02/2019

Aprovado em 12/07/2019

\section{Como referenciar esse artigo}

BIANCHINI, Sara. Kant e la Rivoluzione Francese: liberal e/o reazionari fra passione e storia. Argumentos: Revista de Filosofia. Fortaleza, ano 11, n. 22, p. 72-90, jul.-dez. 2019. 